\title{
PROGRESIÓN DE ENFERMEDAD RENAL CRÓNICA EN UN HOSPITAL DE REFERENCIA DE LA SEGURIDAD SOCIAL DE PERÚ 2012-2015
}

\author{
Jessica Bravo-Zúñiga ${ }^{1,2, a}$, Ricardo Chávez-Gómez ${ }^{1, a}$, Jungmei Gálvez-Inga1,a ${ }^{1,}$ Mirko Villavicencio-Carranza ${ }^{1, a}$, \\ José Espejo-Sotelo1,a, Manuel Riveros-Aguilar ${ }^{1, a}$
}

\begin{abstract}
RESUMEN
Objetivos. Describir las características de la población con enfermedad renal crónica (ERC) estadio 3 y 4 , determinar los factores asociados a progresión de ERC y a ingreso a terapia de reemplazo renal (TRR), así como la sobrevida renal. Materiales y métodos. Estudio retrospectivo longitudinal de pacientes remitidos entre enero de 2012 y diciembre de 2015 a la Unidad de Salud Renal del Hospital Nacional de Edgardo Rebagliati Martins (HNERM), quienes fueron evaluados y seguidos por un equipo multidisciplinario. Los datos clínicos y de laboratorio de cada consulta se registraron en un software creado específicamente para el programa. Se realizó un análisis de regresión logística multivariado para evaluar los factores asociados con la progresión de la ERC, un modelo de regresión de Cox para predecir el riesgo de ingresar al TRR y el método de Kaplan-Meier para el análisis de supervivencia renal. Resultados. Se evaluó a 1248 pacientes en estadio 3A: 248 (20\%), estadio 3b: 548 (44\%) y estadio 4: $452(36 \%) .352(28 \%)$ progresaron, siendo la proteinuria el factor de progresión más importante (OR: 3,2; IC95\%: 2,2-4,6). La proteinuria incrementa el riesgo de ingreso a la TRR en cuatro veces y el tener una tasa de filtración glomerular $<30 \%$ en 3,6 veces. La mediana de seguimiento fue de 12 meses (RIC 5-27 meses). 92 pacientes (7\%) requirieron iniciar TRR. La supervivencia renal a los 12 meses de seguimiento fue del $96 \%$ y a los 24 meses de $90 \%$. Conclusiones. Nuestro estudio muestra que en un centro especializado una proporción significativa de pacientes con ERC no progresa en su enfermedad y que el factor que más se asocia a progresión de enfermedad y a inicio de TRR es la proteinuria.
\end{abstract}

Palabras clave: Enfermedad renal crónica; Supervivencia, Progresión de la enfermedad; Perú (fuente: DeCS BIREME).

\section{PROGRESSION OF CHRONIC RENAL DISEASE IN A REFERENCE HOSPITAL OF SOCIAL SECURITY OF PERU 2012-2015}

\begin{abstract}
Objectives. To describe the characteristics of the population with chronic kidney disease (CKD) stage 3 and 4 , to determine the factors associated with CKD progression and admission to renal replacement therapy (RRT), as well as renal survival. Materials and methods. Longitudinal retrospective study of patients referred between January 2012 and December 2015 to the Renal Health Unit of the Hospital Nacional Edgardo Rebagliati Martins (HNERM), who were evaluated and followed by a multidisciplinary team. The clinical and laboratory data for each query were recorded in a software created specifically for the program. A multivariate logistic regression analysis was performed to assess the factors associated with the progression of CKD, a Cox regression model to predict the risk of entering RRT and the Kaplan-Meier method for renal survival analysis. Results. We assessed 1248 patients in stage 3A: 248 (20\%), stage 3B: $548(44 \%)$ and stage 4: $452(36 \%) .352(28 \%)$ progressed, being proteinuria the most important progression factor (OR: 3.2; $\mathrm{Cl} 95 \%$ : 2,2-4.6). Proteinuria increases the risk of admission to RRT in four times and having a glomerular filtration rate $<30 \%$ in 3.6 times. Median follow-up was 12 months (RIC 5-27 months). 92 patients (7\%) required to initiate RRT. Renal survival at 12 months of follow-up was $96 \%$ and at 24 months was $90 \%$. Conclusions. Our study shows that in a specialized center a significant proportion of patients with CKD does not progress in their disease and that the factor that is most associated with progression of disease and at the onset of RRT is proteinuria.
\end{abstract}

Key words: Chronic renal disease; Survival, Progression of disease; Peru (source: MeSH NLM).

\footnotetext{
Unidad de Salud Renal, Departamento de Nefrología, Hospital Nacional Edgardo Rebagliati Martins. Lima, Perú

Universidad Peruana Cayetano Heredia. Lima, Perú

Médico nefrólogo

Recibido: 14/11/2016 Aprobado: 24/05/2017 En línea: 28/06/2017
}

Citar como: Bravo-Zúñiga J, Chávez-Gómez R, Gálvez-Inga J, Villavicencio-Carranza M, Espejo-Sotelo J, Riveros-Aguilar M. Progresión de enfermedad renal crónica en un hospital de referencia de la Seguridad Social de Perú 2012-2015. Rev Peru Med Exp Salud Publica. 2017;34(2):209-17. doi: 10.17843/rpmesp.2017.342.2493 


\section{INTRODUCCIÓN}

La enfermedad renal crónica (ERC) es un problema de salud pública que genera múltiples complicaciones en los pacientes, tales como como uremia, enfermedad cardiovascular y muerte prematura y cuyo tratamiento involucra altos costos ${ }^{(1)}$. La prevalencia de pacientes con ERC que reciben diálisis es variable, y el acceso a ella es desigual, con desventaja para los países y poblaciones con menores ingresos ${ }^{(2)}$. La Sociedad Latinoamericana de Nefrología e Hipertensión reporta un aumento en la prevalencia de pacientes con ERC en diálisis por millón de habitantes, de 119 en 1991

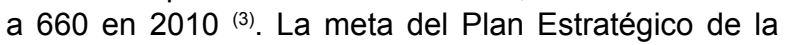
Organización Panamericana de la Salud (OPS) es alcanzar una prevalencia de terapia de reemplazo renal (TRR) mínima de 700 pacientes por millón de habitantes para el 2019. Para lograr esta meta se requiere de un sistema de salud que monitorice y evalúe la progresión de la enfermedad renal, así como impulsar la cobertura universal de servicios de salud y promover acciones de prevención y control de las enfermedades no transmisibles y sus factores de riesgo ${ }^{(4)}$.

Dada su diversidad socioeconómica y geográfica y su rápido crecimiento demográfico la ERC es un problema serio en el Perú y existe una gran demanda de pacientes en estadio 5 (fase terminal) y muy poca oferta de servicios para su tratamiento ${ }^{(5)}$. En el 2013 , el $65 \%$ de la población peruana tenía alguna cobertura de salud ${ }^{(6)}$, sin embargo, solo el $30 \%$ de quiénes necesitaban TRR tenían acceso a esta ${ }^{(7)}$.

En 2011 Francis et al. ${ }^{(8)}$ encontraron una prevalencia de ERC (definida como tasa de filtración glomerular (TFG) estimada $<60 \mathrm{~mL} / \mathrm{min} / 1,73 \mathrm{~m}^{2}$ por fórmula CKD-EPI y una relación proteinuria/ creatinuria $\geq 150 \mathrm{mg} / \mathrm{g}$ ) en poblaciones de Lima y Tumbes de 16,8\% (IC 95\%:13,5\%-20,9\%).

Al no existir datos de estudios nacionales de prevalencia de la ERC en el Perú, Loza ${ }^{(5)}$ en 2016 realizó una aproximación en base a los datos de la encuesta NHANES de los EE.UU., con ello reporta una prevalencia de ERC de $13,2 \%$ y una prevalencia de ERC en diálisis de $0,1 \%$.

Es conocido que la enfermedad renal sin tratamiento, progresa desde formas asintomáticas hasta los estadios finales. Esta progresión puede ser acelerada por factores no modificables (sexo, edad, raza) ${ }^{(9)}$, pero también por factores modificables (proteinuria) ${ }^{(10)}$. Por lo tanto, es importante identificar aquellos factores que puedan predecir mayor rapidez de progresión de la ERC para modificarlos y retrasar la evolución. Se recomienda que las estrategias para retrasar la progresión de la enfermedad renal deben involucrar a un equipo multidisciplinario e incluir un programa educativo para los pacientes ${ }^{(11)}$.

\section{MENSAJES CLAVE}

Motivación para realizar el estudio. El control de los factores asociados a la progresión de la enfermedad renal crónica (ERC) $\mathrm{y}$ al ingreso en terapia de reemplazo renal mejora el curso de la enfermedad. Además, es un tema poco estudiado en Perú.

Principales hallazgos. Se evaluaron 1248 pacientes con ERC estadios 3 y 4 . El $28 \%$ de ellos progresó. La proteinuria mayor de 1,5 g/día fue el principal factor asociado a la progresión y al ingreso a terapia de reemplazo renal. Entre más tarde se deriva al especialista, el riesgo de llegar a diálisis se incrementa.

Implicancias. La proteinuria debe incluirse siempre en el seguimiento clínico de los pacientes con ERC, e insistir en la referencia temprana desde la atención primaria.

El referir a un paciente con enfermedad renal de manera oportuna a un nefrólogo, permite un inicio programado de TRR y disminuye su probabilidad de morir ${ }^{(12)}$. Asimismo, el momento y la calidad del manejo prediálisis y el manejo eficiente de las complicaciones de la uremia, son factores determinantes en la morbimortalidad y calidad de vida de los pacientes ${ }^{(13)}$.

Estudiar los factores asociados a la progresión de ERC en la población peruana es fundamental para diseñar estrategias preventivas adaptadas a nuestro contexto y así contribuir a reducir la morbimortalidad y los costos del sistema de salud.

Por ello, llevamos a cabo un estudio longitudinal retrospectivo para describir las características de la población con ERC en estadio 3 y 4 , atendida en el Hospital Nacional Edgardo Rebagliati Martins (HNERM), determinar los factores asociados a progresión de ERC y a ingreso a TRR, así como la sobrevida renal.

\section{MATERIALES Y MÉTODOS}

Este estudio longitudinal retrospectivo fue llevado a cabo en la Unidad de Salud Renal del Departamento de Nefrología del HNERM, el cual es el hospital más grande de la Seguridad Social de Perú (EsSalud) y atiende al 16\% de la población asegurada (1 707000 personas). Además, cuenta con un equipo multidisciplinario que maneja a pacientes con enfermedad renal siguiendo guías de práctica clínica institucionales ${ }^{(14)}$. La población de estudio incluyó pacientes derivados de los servicios de atención primaria de la Red Asistencial del HNERM, con diagnóstico de ERC, los datos fueron recolectados entre enero de 2012 y diciembre de 2015.

Los médicos de atención primaria efectuaron el tamizaje de ERC (TFG calculada por fórmula de Modification Dialysis Renal Disease 4-MDRD4-y relación albumina/creatinina en orina al azar) en pacientes con factores de riesgo: diabetes, 
hipertensión arterial y/o >55 años. En sus respectivos centros asistenciales, los pacientes con diagnóstico de ERC estadios 3,4 y 5 (TFG calculada $<60 \mathrm{~mL} / \mathrm{min} / 1,73 \mathrm{~m}^{2}$ ) fueron derivados para atención de especialidad.

Para corroborar el diagnóstico de ERC en la consulta externa de nefrología se repitió la creatinina y se calculó la TFG usando la fórmula MDR4. Para el estudio se incluyó a pacientes con TFG al ingreso menor a 60 y mayor a $15 \mathrm{~mL} / \mathrm{min} / 1,73 \mathrm{~m}^{2}$ (estadios 3 y 4), se excluyó a pacientes que podrían desarrollar insuficiencia renal aguda (glomerulonefritis extracapilares, vasculitis o nefropatía lúpica), pacientes con deterioro de la función renal en los tres meses previos debido a nefrotoxicidad, deshidratación, infección o cirugía o que tuvieron menos de dos visitas a nuestras consultas, bien por abandono del seguimiento o por ingreso a TRR sin seguimiento en la unidad.

Los pacientes fueron evaluados y seguidos por un equipo multidisciplinario constituido por un nefrólogo, una enfermera y una nutricionista. El seguimiento ocurría cada tres meses, con el objetivo terapéutico de conseguir un control adecuado de la presión arterial (presión arterial objetivo $<140 / 90 \mathrm{mmHg}$ y en caso de presencia de proteinuria, por debajo de 130/80 $\mathrm{mmHg}$ ) y la corrección de las alteraciones metabólicas, lipídicas y de la anemia. Las nutricionistas recomendaban una restricción moderada de proteínas en la dieta (proteínas: 0,8 a 1g/kg/día), y una dieta personalizada según el índice de masa corporal (IMC) y el estadio de enfermedad. Los pacientes con una hemoglobina inferior a $10 \mathrm{~g} / \mathrm{dL}$, y con valores adecuados de reserva de fierro (saturación de transferrina de 20 a $50 \%$ y ferritina de 200 a $500 \mathrm{ng} / \mathrm{mL}$ ) que no presentaban anemia de otra causa fueron tratados con eritropoyetina subcutánea y fierro oral o endovenoso.

\section{VARIABLES}

La información de cada consulta se registró en el software UMERC, base de datos creada específicamente para el programa de salud renal, de donde se extrajo las variables estudiadas. Se describieron los datos demográficos: sexo, edad y etiología de la enfermedad renal.

Se registró la presión arterial $(\mathrm{mmHg})$ en cada evaluación, hemoglobina $(\mathrm{g} / \mathrm{dL})$ (definiéndose anemia a un valor de hemoglobina $\mathrm{Hb}<10) \mathrm{g} / \mathrm{dL}$, glucosa $(\mathrm{mg} / \mathrm{dl})$, calcio $(\mathrm{mg} /$ $\mathrm{dl}$, fosforo(mg/dL), albúmina $(\mathrm{g} / \mathrm{dL})$, colesterol(mg/dL), triglicéridos $(\mathrm{mg} / \mathrm{dl})$, proteinuria en orina de 24 horas (g/dia), creatinina sérica $(\mathrm{mg} / \mathrm{dl})$, TFG calculada mediante la fórmula de MDRD4 (TFG en $\mathrm{mL} / \mathrm{min}$ por $1,73 \mathrm{~m}^{2}=175$ $\times$ creatinina sérica $^{-1,154} \times$ edad $^{-0,203} \times 1,212$ (si el paciente es de raza negra), $\times 0,742$ (si el paciente es mujer).

También se consideró el uso de algunos medicamentos: uso de eritropoyetina (EPO), uso de inhibidores de enzima convertidora de angiotensina (IECAS), y uso de inhibidores de receptor de angiotensina (ARA2).
Los exámenes auxiliares se procesaron en el laboratorio del HNERM. Para la medición de la creatinina se usó el método de Jaffe; para la medición de la proteinuria se solicitó y se dio las indicaciones para la recolección de orina de 24 horas.

Los estadios de enfermedad renal fueron clasificados de la siguiente manera: $3 a \quad\left(T F G<60 \mathrm{~mL} / \mathrm{min} / 1,73 \mathrm{~m}^{2}\right.$ y $>45 \mathrm{~mL} / \mathrm{min} / 1,73 \mathrm{~m}^{2}$ ), 3b (TFG<45 $\mathrm{mL} / \mathrm{min} / 1,73 \mathrm{~m}^{2}$ y $\left.>30 \mathrm{~mL} / \mathrm{min} / 1,73 \mathrm{~m}^{2}\right)$ y $4\left(\mathrm{TFG}<30 \mathrm{~mL} / \mathrm{min} / 1,73 \mathrm{~m}^{2}\right.$ y $>15$ $\left.\mathrm{mL} / \mathrm{min} / 1,73 \mathrm{~m}^{2}\right)^{(15)}$

La progresión de la enfermedad renal se determinó mediante la diferencia entre el valor de la TFG al final del periodo de seguimiento y la TFG al ingreso al programa (usando la fórmula MDRD4), dividiendo entre el tiempo de seguimiento individual expresado en días, se consideró como progresión: a la pérdida de función renal en 1 año de $>5 \mathrm{~mL} / \mathrm{min} / 1,73 \mathrm{~m}^{2}$; estabilización cuando la TFG varía entre $\pm 5 \mathrm{~mL} / \mathrm{min} / 1,73 \mathrm{~m}^{2}$ en 1 año y regresión cuando la TFG mejoraba en $>5 \mathrm{~mL} / \mathrm{min} / 1,73 \mathrm{~m}^{2}$ en 1 año ${ }^{(16)}$.

La sobrevida renal se definió como el tiempo en meses desde el ingreso del paciente a la unidad de salud renal como fecha inicial y el ingreso a TRR como fecha final, en el periodo de estudio. El paciente ingresaba a TRR cuando presentaba una TFG $<15 \mathrm{~mL} / \mathrm{min} / 1,73 \mathrm{~m}^{2}$ asociado a hallazgos clínicos de uremia, el dato fue verificado por medio de la historia clínica de diálisis.

\section{ANÁLISIS ESTADÍSTICO}

El análisis de datos se realizó en el software estadístico Stata 13. Las variables continuas se presentaron como mediana más rango intercuartil (RIC) ya que tenían distribución sesgada. Las variables categóricas se presentaron como frecuencias y porcentajes. Para comparar variables continuas se usó la prueba $U$ de Mann Whitney. Para comparar variables categóricas se usó la prueba de chi cuadrado. Se definió progresión como variable dependiente, se calculó el odds ratio (OR) y el intervalo de confianza del 95\% (IC 95\%) para las variables predictores potenciales de progresión, donde se incluyó: edad, sexo, etiología de enfermedad, estadio de enfermedad, proteinuria, y anemia; como variables de control: hipoalbuminemia, uso de EPO, uso de ARA2 y uso de IECAS. Luego se realizó el análisis multivariado para definir el modelo asociado a progresión renal en la población estudiada. Se incluyó a las variables que tuvieron un $p<0,2$ (valor utilizado por convención) en el análisis bivariado y a las que se consideró biológicamente relevantes; finalmente, se midió la significancia del modelo.

Se analizaron las características de los pacientes que ingresaron a TRR, mediante un análisis bivariado. Se realizó un modelo multivariado de regresión de Cox para predecir el riesgo de ingresar a TRR. Luego se usó el modelo de Kaplan-Meier y el estadístico log-rank para describir la sobrevida renal del paciente. 
El protocolo fue aprobado por el Comité de Ética del Hospital Nacional Edgardo Rebagliati Martins.

\section{RESULTADOS}

\section{DESCRIPCIÓNDE LAPOBLACIÓN YESTADIOS DE ERC}

En el periodo de estudio, 1836 pacientes ingresaron a la Unidad de Salud Renal. De ellos, 1248 cumplían con los criterios de inclusión. Se excluyó a $588(32,0 \%)$ pacientes: 104(5,7\%) tenían indicación de TRR de urgencia al momento del diagnóstico (TFG $<15 \mathrm{~mL} / \mathrm{min} / 1,73 \mathrm{~m}^{2}$ y síntomas de uremia), $129(7,0 \%)$ tenían TFG $>60 \mathrm{~mL} / \mathrm{min} / 1,73 \mathrm{~m}^{2}$, y 355 $(19,3 \%)$ no tenían datos de seguimiento completos.

De los pacientes estudiados, la mediana de edad era 77 años (RIC 69-84); 304(24,4\%) eran menores de 69 años y $728(58,3 \%)$ eran varones. La etiología más frecuente de ERC fue la hipertensión arterial con $1026(82,2 \%)$ pacientes.
Respecto a los estadios de enfermedad, encontramos: 248 $(19,9 \%)$ pacientes en estadio $3^{\mathrm{a}} ; 548(43,9 \%)$ pacientes en estadio $3 B$ y $452(36,2 \%)$ pacientes en estadio 4 . Respecto a la medicación nefroprotectora: $539(43,2 \%)$ pacientes utilizaban IECAS y $871(69,8 \%)$ usaban ARA2.

Los pacientes que presentaron progresión fueron discretamente más jóvenes: mediana de 76 años (RIC: 67-83) vs. 78 años (RIC: 70-85), de sexo masculino, tenían menor nivel de hemoglobina, albúmina más baja, mayor proteinuria y utilizaban más frecuentemente IECAS. De otro lado, los que no progresan tuvieron valores de proteinuria más bajo con una mediana de 0,2 (RIC: $0,1-0,5)$, y $149(60,1 \%)$ pertenecían al estadio 3 .

Las características demográficas, clínicas y los parámetros de laboratorio de los participantes del estudio en general y según la progresión de ERC se detallan en la Tabla 1.

Tabla 1. Características demográficas, clínicas y de laboratorio de los participantes según la progresión de enfermedad renal crónica (ERC), Unidad de Salud Renal, Hospital Nacional Edgardo Rebagliati Martins, 2012-2015

\begin{tabular}{|c|c|c|c|c|}
\hline Características & $\begin{array}{c}\text { Total } \\
(n=1248)\end{array}$ & $\begin{array}{l}\text { No progresión de ERC } \\
\qquad(n=896)\end{array}$ & $\begin{array}{l}\text { Progresión de ERC } \\
(n=352)\end{array}$ & Valor $p$ \\
\hline Edad en años, med(RIC) & $77(69-84)$ & $78(70-85)$ & $76(67-83)$ & $0,001^{*}$ \\
\hline Sexo masculino, n(\%) & $728(58,3)$ & $529(72,7)$ & $199(27,3)$ & $0,419^{\dagger}$ \\
\hline \multicolumn{5}{|l|}{ Etiología de la ERC, $n(\%)$} \\
\hline Hipertensión arterial & $1026(82,2)$ & $746(72,7)$ & $280(27,3)$ & $0,123^{\dagger}$ \\
\hline Diabetes mellitus & $177(14,2)$ & $120(67,8)$ & $57(32,2)$ & $0,202^{\dagger}$ \\
\hline Enfermedades glomerulares & $26(2,1)$ & $16(61,5)$ & $10(38,5)$ & $0,240^{\dagger}$ \\
\hline Uropatía obstructiva & $19(1,5)$ & $14(76,7)$ & $5(23,3)$ & $0,854^{\dagger}$ \\
\hline \multicolumn{5}{|l|}{ Estadios de ERC n(\%) } \\
\hline Estadio 3A & $248(19,9)$ & $149(60,1)$ & $99(39,9)$ & $<0,001^{\dagger}$ \\
\hline Estadio 3B & $548(43,9)$ & $415(75,7)$ & $133(24,3)$ & $0,006^{\dagger}$ \\
\hline Estadio 4 & $452(36,2)$ & $332(73,5)$ & $120(26,5)$ & $0,327^{\dagger}$ \\
\hline PAM (mmHg) med(RIC) & $96,7(93,3-103,3)$ & $96,7(93,3-103,3)$ & $96,7(93,3-103,3)$ & 0,039 * \\
\hline \multicolumn{5}{|l|}{ Laboratorio, med(RIC) } \\
\hline Hemoglobina (g/dL) & $11,2(10,2-12,4)$ & $11,3(10,2-12,5)$ & $11,0(10,0-12,2)$ & $0,003^{*}$ \\
\hline Fósforo (mg/dL) & $3,7(3,2-4,2)$ & $3,6(3,2-4,1)$ & $3,8(3,3-4,3)$ & $0,038^{*}$ \\
\hline Calcio (mg/dL) & $8,9(8,2-9,3)$ & $8,9(8,3-9,4)$ & $8,8(7,8-9,3)$ & $0,053^{*}$ \\
\hline Albúmina $(\mathrm{g} / \mathrm{dL})$ & $4(3,8-4,3)$ & $4,1(3,8-4,3)$ & $4,0(3,7-4,2)$ & $0,000^{*}$ \\
\hline Colesterol (mg/dL) & $178(152-210)$ & $176(150,5-207)$ & $183(155-214)$ & $0,010^{*}$ \\
\hline Triglicéridos (mg/dL) & 139(99-180) & $137(98-179)$ & $144(104-187)$ & $0,099^{*}$ \\
\hline Proteinuria (g/día) & $0,2(0,1-0,7)$ & $0,2(0,1-0,5)$ & $0,3(0,2-1,3)$ & $<0,001^{*}$ \\
\hline Uso de EPO n(\%) & & & & $0,005^{\dagger}$ \\
\hline Sí & $246(19,7)$ & $159(64,6)$ & $87(35,4)$ & \\
\hline No & $1002(80,3)$ & $737(73,5)$ & $265(26,5)$ & \\
\hline Uso de ARA2 n(\%) & & & & $0,386^{\dagger}$ \\
\hline Sí & $871(69,8)$ & $619(71,1)$ & $252(28,9)$ & \\
\hline No & $377(30,2)$ & $277(73,5)$ & $100(26,5)$ & \\
\hline Uso de IECAS n(\%) & & & & $0,016^{\dagger}$ \\
\hline Sí & $539(43,2)$ & $368(68,3)$ & $171(31,7)$ & \\
\hline No & $709(56,8)$ & $528(74,5)$ & $181(25,5)$ & \\
\hline
\end{tabular}

med: mediana, RIC: rango intercuartilico, ERC: enfermedad renal crónica, PAM: presión arterial media, EPO: eritropoyetina, ARA2: antagonistas de los receptores de angiotensina 2, IECAS: inhibidor de la enzima convertidora de angiotensina

* prueba de suma de rangos ${ }^{\dagger}$ prueba de chi cuadrado 


\section{PROGRESIÓN DE ERC Y FACTORES ASOCIADOS A LA PROGRESIÓN}

En el periodo de estudio, 352(28,2\%) pacientes presentaron progresión de la enfermedad; la mediana de la variación de la TFG fue de -11,9 (RIC: -7,2 a -20,2) $\mathrm{mL} / \mathrm{min} /$ año; $499(39,9 \%)$ pacientes se mantuvieron estables y $397(31,9 \%)$ pacientes mejoraron. La variación en la TFG de los que llegaron en estadio $3 A$ fue de $-2,4$

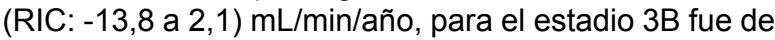
0 (RIC: $-4,5$ a 4,2$) \mathrm{mL} / \mathrm{min} /$ año y para el estadio 4 fue de $-0,3$ (RIC: $-5,4$ a 2,5) $\mathrm{mL} / \mathrm{min} /$ año.

La enfermedad renal progresó en $99(39,9 \%)$ de los pacientes que llegaron en estadio $3 A$, en $133(24,3 \%)$ de los pacientes que llegaron en estadio $3 B$, y en $120(26,6 \%)$ de los que llegaron en estadio 4.

Al realizar la regresión logística multivariada, se encontró que el modelo de paciente que presenta mayor progresión de ERC es aquel con proteinuria $>1,5 \mathrm{~g} /$ día, con TFG entre 45 y $60 \mathrm{~mL} / \mathrm{min} / 1,73 \mathrm{~m}^{2}$, que presenta hipoalbuminemia y usa IECAS. Sin embargo, fue la proteinuria $>1,5 \mathrm{~g} /$ día (OR: 3,$2 ;$ IC: 2,2-4,6) el factor más importante para la progresión tal como se aprecia en la Tabla 2.

\section{FACTORES ASOCIADOS A INGRESO A TRR Y DETERMINANTES DE SOBREVIDA RENAL}

La tabla 3 muestra los resultados más resaltantes respecto al análisis bivariado asociado a ingreso a TRR, los pacientes que llegan a esta terapia son habitualmente más jóvenes <69 años (HR 2,9; IC95\% 1,6-5,3), utilizan con más frecuencia EPO (HR 3,1; IC95\% 2,1-4,7), además tienen hipoalbuminemia (HR 4,5; IC95\% 2,7-7,2), proteinuria $>1,5 \mathrm{~g} /$ día y son aquellos que ingresaron a la unidad en estadio 4. Lo más destacable del análisis multivariado es que la proteinuria>1,5 g/día aumenta el

Tabla 2. Análisis logístico bivariado y multivariado de las características basales asociadas a progresión de enfermedad renal crónica (ERC) en 1248 pacientes de la Unidad de Salud Renal, Hospital Nacional Edgardo Rebagliati Martins, 2012-2015

\begin{tabular}{|c|c|c|c|c|}
\hline Características & $\begin{array}{c}\text { No progresión de ERC } \\
(n=896,71,8 \%)\end{array}$ & $\begin{array}{c}\text { Progresión de ERC } \\
(\mathrm{n}=352,28,2 \%)\end{array}$ & OR crudo (IC95\%) & 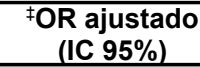 \\
\hline Sexo & $n(\%)$ & $\mathrm{n}(\%)$ & & \\
\hline Masculino & $529(72,7)$ & $199(27,3)$ & $0,90(0,70-1,15)$ & - \\
\hline Femenino & $367(70,6)$ & $153(29,4)$ & 1 & \\
\hline \multicolumn{5}{|l|}{ Edad } \\
\hline$<69$ años & $214(64,7)$ & $117(35,3)$ & 1 & \\
\hline 69-77 años & $212(71,6)$ & $84(28,4)$ & $0,72(0,51-1,01)$ & - \\
\hline 78-84 años & $240(75,0)$ & $80(25,0)$ & $0,61(0,43-0,85)$ & - \\
\hline$>84$ años & $230(71,8)$ & $71(28,2)$ & $0,56(0,40-0,80)$ & - \\
\hline \multicolumn{5}{|l|}{ Etiología } \\
\hline Uropatia obstructiva & $14(73,7)$ & $5(26,3)$ & 1 & \\
\hline Hipertensión arterial & $746(72,7)$ & $280(27,3)$ & $1,05(0,37-2,94)$ & - \\
\hline Diabetes mellitus & $120(67,8)$ & $57(32,2)$ & $1,33(0,45-3,88)$ & - \\
\hline Enfermedades glomerulares & $16(61,5)$ & $10(38,5)$ & $1,75(0,46-6,52)$ & - \\
\hline \multicolumn{5}{|l|}{ Estadio de enfermedad } \\
\hline Estadio 3A & $149(60,1)$ & $99(39,9)$ & $1,96(1,47-2,62)$ & $2,17(1,57-3,02)$ \\
\hline Estadio 3B & $415(75,7)$ & $133(24,3)$ & $0,70(0,55-0,91)$ & $1,02(0,73-1,43)$ \\
\hline Estadio 4 & $332(73,5)$ & $120(26,5)$ & 1 & 1 \\
\hline \multicolumn{5}{|l|}{ Hemoglobina (gr/dL) } \\
\hline No anemia & $551(73,3)$ & $201(26,7)$ & 1 & \\
\hline Anemia* & $286(68,4)$ & $132(31,6)$ & $1,26(0,97-1,64)$ & - \\
\hline \multicolumn{5}{|l|}{ Albumina (gr/dL) } \\
\hline No hipoalbuminemia & $735(72,9)$ & $273(27,1)$ & 1 & 1 \\
\hline Hipoalbuminemia & $46(53,5)$ & $40(46,5)$ & $2,34(1,50-3,65)$ & $2,11(1,31-3,39)$ \\
\hline \multicolumn{5}{|l|}{ Proteinuria(g/día) } \\
\hline$<0,30$ & $552(76)$ & $174(24)$ & 1 & 1 \\
\hline$>0,3 y<1,5$ & $248(71,9)$ & $97(28,1)$ & $1,24(0,92-1,65$ & $1,10(0,79-1,53)$ \\
\hline$>1,5$ & $80(51,6)$ & $75(48,4)$ & $2,97(2,06-4,29)$ & $3,17(2,18-4,61)$ \\
\hline No uso de EPO & $737(73,6)$ & $265(26,4)$ & 1 & \\
\hline Uso de EPO & $159(64,6)$ & $87(35,4)$ & $1,52(1,13-2,04)$ & - \\
\hline No uso de ARA2 & $277(73,5)$ & $100(26,5)$ & 1 & \\
\hline Uso de ARA2 & $619(71,1)$ & $252(28,9)$ & $1,12(0,86-1,48)$ & \\
\hline No uso de IECAS & $528(74,5)$ & $181(25,5)$ & 1 & 1 \\
\hline Uso de IECAS & $368(68,3)$ & $171(31,7)$ & $1,35(1,05-1,74)$ & $1,43(1,08-1,88)$ \\
\hline
\end{tabular}

OR: Odds Ratio, IC: intervalo de confianza, EPO: Eritropoyetina ARA2: antagonistas de los receptores de angiotensina 2, IECAS: Inhibidor de la enzima convertidora de angiotensina

*Se definió anemia cuando el valor de hemoglobina $<10 \mathrm{gr} / \mathrm{dL}$

† Se definió hipoalbuminemia cuando el valor de albumina en sangre $<3,0 \mathrm{gr} / \mathrm{dL}$

¥Ajustado por todas las variables, se incluyó a las variables que tuvieron un $p<0,2$ en el modelo crudo y a las que se consideró biológicamente relevantes 
riesgo de ingreso a hemodiálisis en cuatro veces (HR 4; IC95\% 1,3-6,3).

La mediana del seguimiento de los pacientes fue de 12,4 meses (RIC 5,1-27,2). Durante el periodo de seguimiento, 92 pacientes $(7,4 \%)$ requirieron iniciar TRR.
La sobrevida renal a los 12 meses de seguimiento fue de $95,5 \%($ IC $95 \% 93,9-96,7)$ y a los 24 meses de seguimiento de $89,9 \%(I C 95 \% 87,9-92,0)$. Tal como se muestra en la figura 1 existe una diferencia estadísticamente significativa en la sobrevida renal de los pacientes que progresan versus aquellos que no lo hacen $(p<0,01)$.

Tabla 3. Modelos de riesgos proporcionales de Cox bivariado y multivariado de las características basales asociadas a ingreso a terapia de reemplazo renal (TRR) en 1248 pacientes en la Unidad de Salud Renal, Hospital Nacional Edgardo Rebagliati Martins, 2012-2015

\begin{tabular}{|c|c|c|c|c|}
\hline Características & $\begin{array}{c}\text { No ingresó a TRR } \\
(\mathrm{n}=1156,92,6 \%)\end{array}$ & $\begin{array}{c}\text { Si ingreso a TRR } \\
(\mathrm{n}=92,7,4 \%)\end{array}$ & HR Crudo (IC95\%) & $\begin{array}{l}\text { ‡HR Ajustado } \\
\text { (IC95\%) }\end{array}$ \\
\hline Sexo & $\mathrm{n}(\%)$ & $\mathrm{n}(\%)$ & & \\
\hline Masculino & $673(92,5)$ & $55(7,5)$ & $0,97(0,64-1,47)$ & - \\
\hline Femenino & $483(92,9)$ & $37(7,1)$ & 1 & \\
\hline Edad(años) & & & & - \\
\hline$<69$ & $292(88,2)$ & $39(11,8)$ & $2,90(1,57-5,35)$ & - \\
\hline $69-77$ & $274(92,6)$ & $22(7,4)$ & $1,83(0,93-3,57)$ & - \\
\hline $77-84$ & $303(94,7)$ & $17(5,3)$ & $1,08(0,53-2,20)$ & - \\
\hline$>84$ & $287(95,4)$ & $14(4,6)$ & 1 & \\
\hline \multicolumn{5}{|l|}{ Etiología } \\
\hline Hipertensión arterial & $960(93.6)$ & $66(6.4)$ & $1,03(0,30-3,49)$ & - \\
\hline Diabetes mellitus tipo 2 & $159(89,8)$ & $18(10,2)$ & $0,59(0,35-1,05)$ & - \\
\hline Glomerular* & $21(80,8)$ & $5(19,2)$ & $1,81(0,67-4,90)$ & - \\
\hline Posrenal** $^{* *}$ & $16(84,2)$ & $3(15,8)$ & 1 & \\
\hline \multicolumn{5}{|l|}{ Estadio de enfermedad } \\
\hline Estadio 3A & $247(99,6)$ & $1(0,4)$ & 1 & \\
\hline Estadio 3B & $526(96,0)$ & $22(4,0)$ & $8,63(1,16-64,09)$ & $7,55(0,99-56,06)$ \\
\hline Estadio 4 & $383(84,7)$ & $69(15,3)$ & $19,38(2,67-140,55)$ & $3,57(2,18-5,85)$ \\
\hline \multicolumn{5}{|l|}{ Hemoglobina (g/dL) } \\
\hline No anemia & $707(94,0)$ & $45(6,0)$ & 1 & \\
\hline Anemia & $371(88,8)$ & $47(11,2)$ & $0,94(0,47-1,87)$ & - \\
\hline \multicolumn{5}{|l|}{ Albumina (g/dL) } \\
\hline No hipoalbuminemia & $940(93,2)$ & $68(6,8)$ & 1 & \\
\hline Hipoalbuminemia & $64(74,4)$ & $22(25,6)$ & $4,45(2,75-7,20)$ & $2,07(1,19-3,47)$ \\
\hline \multicolumn{5}{|l|}{ Proteinuria (g/día) } \\
\hline$<0,30$ & $709(97,7)$ & $17(2,3)$ & 1 & \\
\hline$>0,3 y>1,5$ & $315(91,3)$ & $30(8,7)$ & $3,71(1,05-6,75)$ & $2,89(0,99-5,37)$ \\
\hline$>1,5$ & $110(71,0)$ & $45(29,0)$ & $13,65(7,81-23,86)$ & $4,05(1,30-6,33)$ \\
\hline Uso de ARA2 & $804(92,3)$ & $67(7,7)$ & $1,20(0,75-1,90)$ & - \\
\hline No uso de ARA2 & $352(93,4)$ & $25(6,6)$ & 1 & \\
\hline Uso de IECAS & $494(91,7)$ & $45(8,3)$ & $1,28(0,85-1,96)$ & - \\
\hline No uso de IECAS & $662(93,4)$ & $47(6,6)$ & 1 & \\
\hline Uso de EPO & $209(85,0)$ & $37(15,0)$ & $3,11(2,05-4,71)$ & $2,01(1,30-3,09)$ \\
\hline No uso de EPO & $947(94,5)$ & $55(5,5)$ & 1 & \\
\hline
\end{tabular}

HR: Hazard Ratio, EPO: eritropoyetina ARA2: antagonistas de los receptores de angiotensina 2, IECAS: inhibidor de la enzima convertidora de angiotensina *Se definió anemia cuando el valor de hemoglobina $<10 \mathrm{gr} / \mathrm{dL}$

+ Se definió hipoalbuminemia cuando el valor de albumina en sangre $<3,0 \mathrm{gr} / \mathrm{dL}$

${ }_{\ddagger}$ Ajustado por todas las variables, se incluyó a las variables que tuvieron un $\mathrm{p}<0,2$ en el modelo crudo y a las que se consideró biológicamente relevantes 


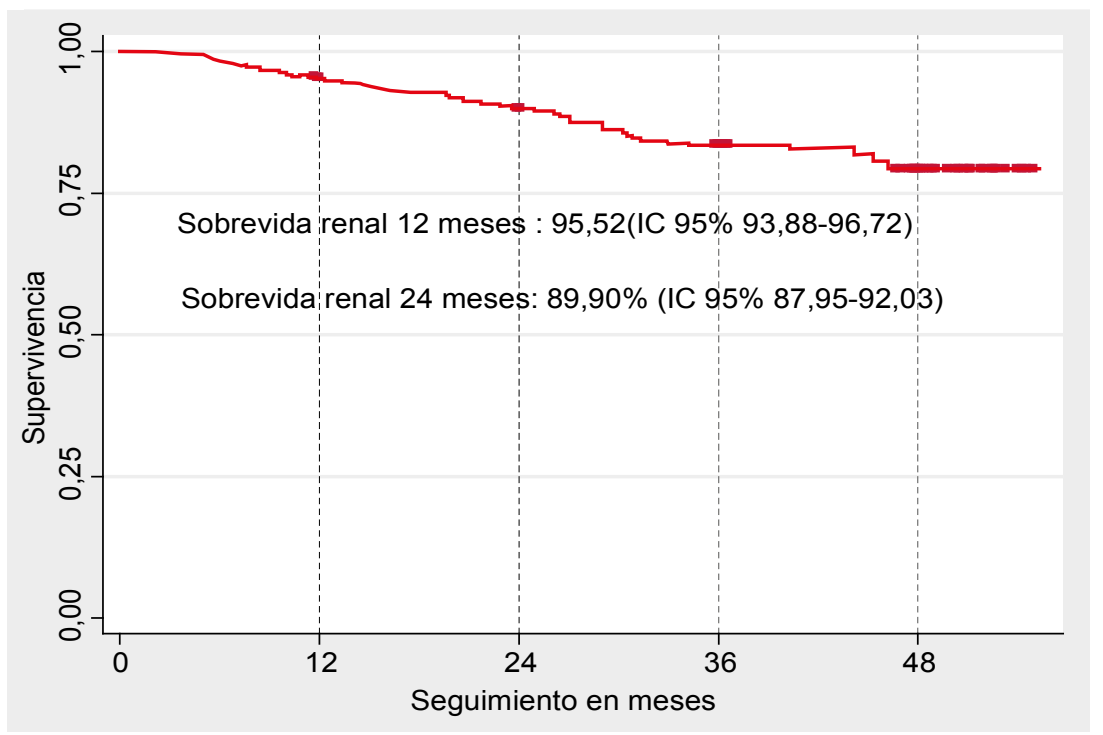

Figura 1. Curva de Kaplan-Meier para evaluar sobrevida renal en 1248 pacientes en la Unidad de Salud Renal, Hospital Nacional Edgardo Rebagliati 2012-2015

\section{DISCUSIÓN}

Los principales hallazgos de este estudio muestran que más de un cuarto de los pacientes evaluados presenta progresión de la ERC. Estos pacientes son aquellos que tienen hipoalbuminemia, probablemente en relación a la pérdida urinaria de proteínas, usan IECAS, tienen una función renal entre 45 y $60 \mathrm{~mL} / \mathrm{min} / 1,73 \mathrm{~m}^{2}$, pero principalmente, presentan mayor proteinuria.

Múltiples estudios demuestran que el progreso de la ERC, una vez establecida, depende de factores hemodinámicos y metabólicos, independientemente de la causa que condujo a la pérdida de masa renal (17). La progresión de la ERC en el tiempo no es lineal, hay episodios prolongados de progreso lento y episodios en la que la TFG disminuye de manera acelerada ${ }^{(18,19)}$. En nuestro estudio, el $71,8 \%$ de pacientes con ERC se estabilizó o mejoró, lo que puede estar relacionado a la evaluación y seguimiento sistematizado y multidisciplinario que estos pacientes recibieron.

Algo similar se ha encontrado en un centro nefrológico regional en Inglaterra ${ }^{(20)}$ más del $50 \%$ de los pacientes en estadio 3,4 y 5 aún se mantenían en el mismo estadio luego de un año de seguimiento. En Nueva York ${ }^{(21)}$, el $64,3 \%$ de los pacientes referidos en estadio $3 \mathrm{~A}$ y el $27,2 \%$ en $3 \mathrm{~B}$ permanecían en el mismo estadio o habían mejorado en un seguimiento a 10 años. En nuestro estudio, encontramos que $40 \%$ de los pacientes se mantenían en el mismo estadio y el $31,8 \%$ mejoro.

Además, hay que tomar en cuenta las características clínicas de los pacientes que no progresaron: mayores de
69 años, hipertensos y con valores de proteinuria bajos (<0,3 g/día). Varios autores han planteado una relación directa entre la edad y la progresión de ERC. Zhang y Rothenbacher ${ }^{(22)}$ realizaron una revisión sistemática de 26 estudios, para conocer la prevalencia de ERC, y concluyeron que: a) la prevalencia media de ERC es del $7,2 \%$ en la población $<30$ años, mientras que en población anciana oscila entre el 23,4 y $35,8 \%$; b) la prevalencia varía según el método de estimación de TFG utilizado: si se usa la formula MDRD es del $35,8 \%$, si se utiliza la fórmula de Cockcroft-Gault, aumenta a $58,5 \%$, c) Por tanto, la detección exacta de ERC en grupos especiales, particularmente en ancianos, no es exacta.

Entre los pocos estudios específicos realizados en población anciana, cabe nombrar el de Hemmelgarm et al. ${ }^{(23)}$ en el que se describe una población mayor de 66 años y la progresión de la ERC a lo largo de 2 años de seguimiento, siendo esta lenta, excepto en los pacientes diabéticos y en aquellos casos que al inicio del seguimiento tenían una TFG menor a $30 \mathrm{~mL} / \mathrm{min}$.

El hallazgo de la macroalbuminuria como predictor de rápida progresión de ERC también se ha descrito. En un hospital regional de Escocia ${ }^{(24)}$, la macroalbuminuria fue un predictor de progresión de ERC, así como de menor sobrevida renal. Levin et al. (25) encontraron que los pacientes que más progresan son los que se encuentran en menor estadio siempre y cuando además tengan cifras altas de proteinuria.

Debido a que la progresión de ERC es muy variable, las Guías KDIGO sugieren que si no se puede identificar a quiénes tendrán una progresión rápida, es recomendable 
evaluar simultánea y sistemáticamente la TFG estimada y la albuminuria, ambos parámetros condicionan el pronóstico, ejerciendo, además, un efecto sinérgico ${ }^{(26)}$.

En nuestro estudio encontramos una sobrevida renal de $95,5 \%$ al año y $89,9 \%$ a los dos años. En una cohorte de pacientes en Colombia, con TFG $<30 \mathrm{~mL} / \mathrm{min}{ }^{(27)}$, la sobrevida renal fue de $87 \%$ al año y $76 \%$ a los dos años. En un metaanálisis que incluyo más de 1 millón de pacientes ${ }^{(28)}$, una TFG menor y una mayor proteinuria estuvieron asociadas a menor sobrevida renal, independientemente de otros factores. Por otro lado, Mahdavi-Mazdeh et al. ${ }^{(29)}$ demostraron que los pacientes que son seguidos por un médico nefrólogo tienen mayor sobrevida renal y progresión de ERC más lenta versus aquellos que son seguidos por un médico no especialista.

Entre las limitaciones del estudio tenemos que no se tuvo datos completos de seguimiento para $19,3 \%$ de los pacientes elegibles. Si el pronóstico de estos pacientes fue malo, es posible que hayamos subestimado la progresión renal. Asimismo, no pudimos medir algunas variables potencialmente predictores de progresión de ERC como la dieta del paciente y el nivel de hemoglobina glicosilada. Entre las fortalezas del estudio está que se trata de una población numerosa en el hospital más grande del país, con características específicas en cuanto a edad y etiología de ERC, siendo el primer reporte de seguimiento de pacientes con ERC sin diálisis en nuestro país.
Nuestro estudio muestra que, en un centro especializado, una proporción importante de pacientes con ERC, no progresan a ERC terminal, y que el factor principal asociado a progresión y a ingreso a TRR es la proteinuria. El conocer los determinantes de progresión, permite a los equipos multidisciplinarios detectar a tiempo pacientes que puedan requerir un cuidado más cercano y alerta respecto a la importancia de la remisión temprana al especialista. Replicar a todos los servicios de nefrología del país la experiencia de cuidado multidisciplinario de pacientes con ERC estadio 3-5 podría contribuir a reducir la carga de ERC terminal.

Contribuciones de los autores: JBZ participo en la concepción del artículo, la recolección de datos, el análisis e interpretación de resultados y la redacción del artículo. $\mathrm{RCH}$, JGI, MVC participaron en la recolección de datos, interpretación de resultados y redacción del artículo. JES y MRA realizaron la recolección de datos y redacción del artículo. Todos se encargaron de la revisión crítica y aprobación de la versión final

Agradecimientos: al Instituto de Evaluación de Tecnologías en Salud e Investigación (IETSI) de EsSalud por su apoyo en la elaboración de este manuscrito a través de su Programa de Mentoring y a la Jefatura del Departamento de Nefrología del Hospital Nacional Edgardo Rebagliati por las facilidades para su ejecución.

Fuentes de financiamiento: autofinanciado

Conflictos de interés: ninguno

\section{REFERENCIAS BIBLIOGRÁFICAS}

1. Alcázar R, Egocheaga MI, Orte L, Lobos JM, González Parra E, Álvarez Guisasola F, et al. Documento de consenso SEN-semFYC sobre la enfermedad renal crónica. Nefrología 2008;28(3)273-82.

2. Loza Munarriz C. La enfermedad renal crónica en el Perú, Epidemiologia e impacto de la salud pública (SE) $\mathrm{N}$ $^{\circ}$ 3. Boletín epidemiológico enero 2014. Disponible en: http://www. dge.gob.pe/portal/docs/vigilancia/ boletines/2014/03.pdf

3. Rosa-DiezG,Gonzalez-Bedat M,PecoitsFilho R, Marinovich S, Fernandez S, Lugon $\mathrm{J}$ et al. Renal replacement therapy in Latin American end-stage renal disease. Clin Kidney J. 2014;7(4):431-6. doi: $10.1093 / \mathrm{ckj} / \mathrm{sfu} 039$.

4. Organización Panamericana de la Salud. Plan estratégico de la Orga- nización Panamericana de la Salud 2014-2019[Internet]. Washington DC: OPS; 2013. [citado 22 de octubre de 2016].Disponible en: http:// iris.paho.org/xmlui/bitstream/handle/123456789/4034/OD345-s.pdf ?sequence $=1 \&$ isAllowed $=\mathrm{y}$

5. Loza Munarriz CA, Ramos Muñoz WC. Análisis de la situación de la enfermedad renal crónica en el Perú, 2015 [Internet]. Lima: Dirección General de Epidemiologia, Ministerio de Salud, 2016. Disponible en: http://www. dge.gob.pe/portal/index.php?option $=$ com_content $\&$ view $=$ article $\&$ i $\mathrm{d}=598 \&$ Itemid $=353$

6. Alcalde-Rabanal JE, Lazo-González O, Nigenda G. Sistema de salud de Perú. Salud Publica Mex. 2011;53:S243-54.

7. Dirks JH, Robinson S, Burdmann E, Correa-Rotter R, Mezzano S, Rodriguez Iturbe $\mathrm{B}$. Prevention strategies for chronic kid- ney disease in Latin America: a strategy for the next decade - a report on the Villarica Conference. Ren Fail. 2006;28(8):611-5. doi: 10.1080/08860220600925669.

8. Francis ER, Kuo CC, Bernabe-Ortiz A, Nessel L, Gilman RH, et al. Checkley W. Burden of chronic kidney disease in resource-limited settings from Peru: a population-based study. BMC Nephrology. 2015;16:114. doi:10.1186/s12882-015-0104-7.

9. Remuzzi G, Ruggenti P, Benigni A: Understanding the nature of renal disease progression. Kidney Int. 1997;51(1):2-15.

10. Ruggenenti P, Perna A, Mosconi L, Matalone M, Pisoni R, Gaspari F, et al. Proteinuria predicts end-stage renal failure in non-diabetic chronic nephropathies. The "Gruppo Italiano di Studi Epidemiologici in Nefrologia" (GISEN). Kidney Int. 1997(Supl. 63):S54-7. 
11. Levin A,Hemmelgarn B, Culleton B, Tobe S, McFarlane P, Ruzicka M, et al. Guidelines for the management of chronic kidney disease. CMAJ. 2008;179(11):1154-62. doi: 10.1503/cmaj.080351.

12. Obrador GT, Pereira B. Early referral to the Nephrologist and timely initiation of renal replacement therapy: A paradigm shift in the management of patients with chronic renal failure. Am J Kidney Dis. 1998;31(3):398-417.

13. Baer G, Lameire N, Van Biesen W. Late referral of patients with end-stage renal disease: an in-depth review and suggestions for further actions. NDT Plus. 2010; 3(1):17-27. doi: 10.1093/ndtplus/sfp050.

14. Essalud. Guía de Manejo de Enfermedad Renal Crónica, 2004.

15. National Kidney Foundation. K/DOQI clinical practice guidelines for chronic kidney disease: evaluation, classification, and stratification. Am J Kidney Dis. 2002;39 (2 Supl. 1): S1-266.

16. Borrelli S, Leonardis D, Minutolo R, Chiodini P, De Nicola L, Esposito C, et al. Epidemiology of CKD Regression in Patients under Nephrology Care. PLoS One. 2015;10(10):e0140138. doi: 10.1371/journal.pone.0140138.

17. Gorostidi M, Santamaría R, Alcázar R, Fernández-Fresnedo G, Galcerán $\mathrm{JM}$, Goicoechea M, et al. Documento de la Sociedad Española de Nefrología sobre las guías KDIGO para la evaluación y el tratamiento de la enfermedad renal crónica. Nefrología 2014;34(3):302-16.

18. O'Hare AM, Batten A, Burrows NR, Pavkov ME, Taylor L, Gupta I, et al. Trajectories of kidney function decline in the 2 years before initiation of long-term dialysis. Am J Kidney Dis. 2012;59:513-22. doi: 10.1053/j. ajkd.2011.11.044.
19. Heaf JG, Mortensen LS. Uraemia progression in chronic kidney disease stages $3-5$ is not constant. Nephron Clin Pract. 2011;118(4):c367-74. doi: 10.1159/000323391.

20. Hoefield RA, Kalra PA, Baker P, Lane B, New JP, O'Donoghue DJ, et al. Factors associated with kidney disease progression and mortality in a referred CKD population. Am J Kidney Dis. 2010;56(6):1072-81. doi: 10.1053/j. ajkd.2010.06.010.

21. Baek SD, Baek $\mathrm{CH}$, Kim JS, Kim SM, Kim JH, Kim SB. Does stage III chronic kidney disease always progress to end-stage renal disease? A ten-year follow-up study. Scand J Urol Nephrol. 2012;46(3):232-8. doi: 10.3109/00365599.2011.649045.

22. Zhang QL, Rothenbacher D. Prevalence of chronic kidney disease in population-based studies: systematic review. BMC Public Health. 2008;8:117. doi: 10.1186/1471-24588-117.

23. Hemmelgarn BR, Zhang J, Manns BJ, Tonelli M, Larsen E, Ghali WA, et al. Progression of kidney dysfunction in the community-dwelling elderly. Kidney Int. 2006;69(12):2155-61. doi: 10.1038/sj.ki.5000270.

24. El-Ghoul B, Elie C, Sqalli T, Jungers P, Daudon M, Grünfeld JP, et al. Nonprogressive kidney dysfunction and outcomes in older adults with chronic kidney disease. J Am Geriatr Soc. 2009;57(12):2217-23. doi: 10.1111/j.1532-5415.2009.02561.x.

25. Levin A, Djurdjev O, Beaulieu M, Er L. Variability and risk factors for kidney disease progression and death following attainment of stage $4 \mathrm{CKD}$ in a referred cohort. Am J Kidney Dis. 2008, 52(4):661-71. doi: $10.1053 / \mathrm{j}$. ajkd.2008.06.023.
26. Van der Velde M, Matsushita K, Coresh J, Astor BC, Woodward M, Levey A, et al. Lower estimated glomerular filtration rate and higher albuminuria are associated with allcause and cardiovascular mortality. A collaborative meta-analysis of highrisk population cohorts. Kidney Int 2011;79(12):1341-52. doi: 10.1038/ ki.2010.536.

27. Rodríguez Pabón RE, Nieto $M$, Corrado P, Martínez A, Escobar CM, Jaramillo C. Retardo en la progresión del daño renal en pacientes con insuficiencia renal Crónica estado 4 , impacto de un programa de prevención en prediálisis. Revista Asocolnef. 2007;1(0):16-21.

28. Gansevoort RT, Matsushita K, van der Velde M, Astor BC, Woodward M, Levey AS, et al. Lower estimated GFR and higher albuminuria are associated with adverse kidney outcomes. A collaborative meta-analysis of general and high-risk population cohorts. Kidney Int 2011; 80(1):93-104. doi: 10.1038/ki.2010.531.

29. Mahdavi-Mazdeh M, Nadia Z, Shahpari-Niri S. Does a medical management program for CKD patients postpone renal replacement therapy and mortality? A 5-year-cohort study. BMC Nephrol. 2012;13:138. doi: 10.1186/1471-2369-13-138.

Correspondencia: Jessica Bravo Zuñiga

Dirección: Francisco Graña 674 departamento 204, Lima 17, Perú

Teléfono: (511) 995020905

Correoelectrónico:ivobz@yahoo.es 